PROCEEDINGS OF THE

AMERICAN MATHEMATICAL SOCIETY

Volume 34, Number 2, August 1972

\title{
SUBSEQUENTIAL LIMIT POINTS OF SUCCESSIVE APPROXIMATIONS
}

\author{
L. F. GUSEMAN, JR. AND J. L. SOLOMON
}

\begin{abstract}
Results concerning the structure, cardinality, and invariance of the cluster set of the subsequential limit points of successive approximations are presented. These results along with some examples answer questions posed in a recent paper by $\mathrm{F}$. T. Metcalf and T. D. Rogers.
\end{abstract}

1. Introduction. In a recent paper, [1], Metcalf and Rogers investigated certain properties of the set of subsequential limit points of successive approximations. Motivation for their paper was provided by the study of initial-value problems; in particular, an initial-value problem whose unique solution could not be obtained as the limit of its (Picard) sequence of successive approximations (see the references in [1] for examples). They observed that, even though the sequence of successive approximations need not converge, the sequence may have cluster points (or, subsequential limit points) which give some information about the initial-value problem (such as satisfying a coupled system). Consequently, they studied the behavior of the cluster points under the mapping which defines the successive approximations in the following general setting:

Let $(1)(Y, d)$ be a metric space;

(2) $T$ be a continuous function from $Y$ into $Y$;

(3) $\left\{x_{n}\right\}_{n=0}^{\infty}$ be the sequence of successive approximations, $x_{n+1}=$ $T x_{n}, n=0,1,2, \cdots$, for $x_{0} \in Y$;

(4) $\mathscr{L}$ denote the cluster set (subsequential limit points) of $\left\{x_{n}\right\}_{n=0}^{\infty}$;

(5) $\mathscr{L}^{\prime}$ denote the derived set of $\mathscr{L}$;

(6) $V\left\{x_{n}\right\}_{n=0}^{\infty}$ denote the range of $\left\{x_{n}\right\}_{n=0}^{\infty}$.

It is assumed throughout that $X=V\left\{x_{n}\right\}_{n=0}^{\infty} \cup \mathscr{L}$ is a compact subset of $Y$. Consequently, $\mathscr{L}$ is closed and nonempty, $T \mathscr{L}=\mathscr{L}$, and $\mathscr{L}^{\prime} \subseteq T \mathscr{L}^{\prime}$.

Metcalf and Rogers presented conditions (Theorems I and II below) which insure that $\mathscr{L}^{\prime}=T \mathscr{L}^{\prime}$, and posed the following questions:

A. Does one always have $\mathscr{L}^{\prime}=T \mathscr{L}^{\prime}$ ?

B. Does $\mathscr{L}^{\prime}$ consist only of fixed points?

Received by the editors October 11, 1971.

AMS 1969 subject classifications. Primary 5485, 4785; Secondary 3404.

Key words and phrases. Fixed points, subsequential limit point, successive approximations, cluster set of sequence of successive approximations.

(c) American Mathematical Society 1972 
In $\S 2$ we present an example which answers both $\mathrm{A}$ and $\mathrm{B}$ in the negative. Another example shows that even when $\mathscr{L}^{\prime}=\mathscr{L}, \mathscr{L}^{\prime}$ may contain no fixed points of $T$. Necessary and sufficient conditions that $\mathscr{L}^{\prime}=T \mathscr{L}^{\prime}$, and some results on the cardinality of $\mathscr{L}-\mathscr{L}^{\prime}$ and $\mathscr{L}$ are presented in $\S 3$. Some open problems are discussed in $\S 4$.

The following results from [1] are needed in the sequel.

THEOREM I. Suppose $\mathscr{L}^{\prime} \neq \varnothing$. In the following list of statements, (i) $\Rightarrow$ (ii) $\Rightarrow$ (iii) $\Rightarrow$ (iv).

(i) The set $T^{-1} a$ is finite for each $a \in \mathscr{L}$;

(ii) $T \mathscr{L}^{\prime}=\mathscr{L}^{\prime}$;

(iii) $\mathscr{L}-\mathscr{L}^{\prime} \subseteq T\left(\mathscr{L}-\mathscr{L}^{\prime}\right)$;

(iv) $\mathscr{L}-\mathscr{L}^{\prime}$ is empty or infinite.

THEOREM II. If $\mathscr{L}^{\prime}$ has at most a finite number of nonfixed points of $T$, then $T \mathscr{L}^{\prime}=\mathscr{L}^{\prime}$.

2. Examples. (i) In this example, each of the sets $\mathscr{L}, \mathscr{L}^{\prime}$, and $\mathscr{L}-\mathscr{L}^{\prime}$ is countably infinite, $\mathscr{L}^{\prime}$ is properly contained in $T \mathscr{L}^{\prime}$, and $\mathscr{L}^{\prime}$ contains exactly one fixed point of $T$.

Let $A$ be the set of points on the $x$-axis defined by

$$
A=\{(0,0)\} \cup\{(1 / n, 0): n \geqq 1\} \cup\left\{\left(1 / n+1 / n^{m}, 0\right): n, m \geqq 2\right\} .
$$

The mapping $T$ is defined on a grid of points in the plane as follows:

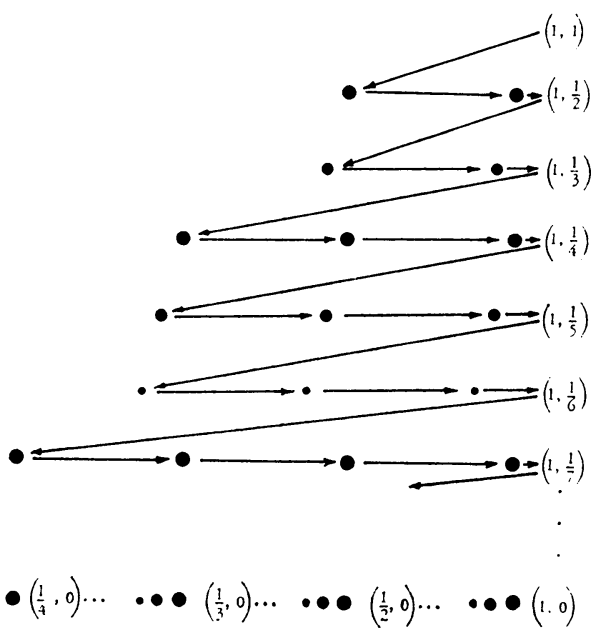

Note that the iterates of $(1,1)$ cover the first $n-1$ terms of the sequence $\left\{\left(1 / n+1 / n^{m}, 0\right)\right\}_{m=2}^{\infty}$ which converges to $(1 / n, 0)$, then proceed to cover the first $n$ terms of the sequence converging to $(1 /(n+1), 0)$. 
For points in $A$, define $T$ as follows:

$$
\begin{aligned}
T(0,0) & =(0,0)=T(1,0), \quad T(1 / 2,0)=(1,0) . \\
T(1 / n, 0) & =(1 /(n-1), 0), \quad n \geqq 3, \\
T\left(1 / n+1 / n^{m}, 0\right) & =\left(1 /(n-1)+1 /(n-1)^{m}, 0\right), \quad n \geqq 3, m \geqq 2, \\
T\left(1 / 2+1 / 2^{m}, 0\right) & =(1,0), \quad m \geqq 2 .
\end{aligned}
$$

Let $Y=A \cup V\left\{T^{n}(1,1)\right\}_{n=0}^{\infty}$. It is easily seen that $T$ is continuous on $Y$, has $(0,0)$ as the only fixed point, and the cluster set of the sequence $\left\{T^{n}(1,1)\right\}_{n=0}^{\infty}$ is given by $\mathscr{L}=A$. Furthermore, we have

$$
\begin{aligned}
\mathscr{L}^{\prime} & =\{(0,0)\} \cup\{(1 / n, 0): n \geqq 2\}, \\
\mathscr{L}-\mathscr{L}^{\prime} & =\{(1,0)\} \cup\left\{\left(1 / n+1 / n^{m}, 0\right): n, m \geqq 2\right\}, \\
T \mathscr{L}^{\prime}-\mathscr{L}^{\prime} & =\{(1,0)\}, \quad \text { and } \\
\mathscr{L}-\mathscr{L}^{\prime} & =T\left(\mathscr{L}-\mathscr{L}^{\prime}\right) .
\end{aligned}
$$

This last set equality shows that the implications (ii) $\Rightarrow$ (iii), (ii) $\Rightarrow$ (iv) of Theorem I cannot be reversed. Note that $T^{-1} a$ is infinite for exactly one point of $\mathscr{L}$, namely $(1,0)$. Also, note that $\mathscr{L}^{\prime}$ has a countably infinite number of nonfixed points of $T$, so that the condition of finiteness in Theorem II cannot be weakened to countable.

(ii) This example illustrates the case in which $\mathscr{L}=\mathscr{L}^{\prime}$ (and thus $\mathscr{L}^{\prime}=$ $T \mathscr{L}^{\prime}$ ) but $\mathscr{L}^{\prime}$ has no fixed points of $T$. Let $Y$ be the subset of the plane defined by (in complex notation)

$$
Y=\left\{z: z=e^{i \theta}, 0 \leqq \theta<2 \pi\right\} .
$$

Let $a$ be irrational, and define $T$ on $Y$ by $T\left(e^{i \theta}\right)=e^{i(\theta+2 \pi a)}$. The cluster set of the sequence $\left\{T^{n}(1,0)\right\}_{n=0}^{\infty}$ is given by $\mathscr{L}=Y=\mathscr{L}^{\prime}, T \mathscr{L}^{\prime}=\mathscr{L}^{\prime}$, but $T$ has no fixed points in $\mathscr{L}$. This example shows that the converse of Theorem II is not true.

3. Main results. Some results concerning the invariance of $\mathscr{L}^{\prime}$ and the cardinality of $\mathscr{L}$ and $\mathscr{L}-\mathscr{L}^{\prime}$ are now presented.

THEOREM 1. Suppose $\mathscr{L}^{\prime} \neq \varnothing$. The following statements are equivalent:

(i) $T \mathscr{L}^{\prime}=\mathscr{L}^{\prime}$;

(ii) $T^{-1} a \cap \mathscr{L}^{\prime}=\varnothing$ for each $a \in \mathscr{L}-\mathscr{L}^{\prime}$;

(iii) $T^{-1} a \cap X$ is finite for each $a \in \mathscr{L}-\mathscr{L}^{\prime}$. (i).

ProOF. (i) $\Rightarrow$ (ii). If $y \in T^{-1} a \cap \mathscr{L}^{\prime}$, then $T y=a \in \mathscr{L}-\mathscr{L}^{\prime}$, contradicting

(ii) $\Rightarrow$ (iii). Let $a \in \mathscr{L}-\mathscr{L}^{\prime}$ and suppose that $y \in T^{-1} a \cap X$ which is infinite. If $y \in V\left\{x_{n}\right\}_{n=0}^{\infty}$ then there exists a positive integer $N$ such that $y=T^{N} x_{0}$. Then since $T \mathscr{L}=\mathscr{L}, T^{N+k} x_{0}=T^{k} y=T^{k-1} a \in \mathscr{L}$ for each $k \geqq 1$. 
It follows that $T^{-1} a \cap \mathscr{L}$ is infinite. Thus there is a sequence $\left\{y_{n}\right\}_{n=0}^{\infty}$ with distinct terms in $T^{-1} a \cap \mathscr{L}$ which converges to $z \in \mathscr{L}^{\prime}$. Since $T^{-1} a$ is closed, $z \in T^{-1} a \cap \mathscr{L}^{\prime}$, which contradicts (ii).

(iii) $\Rightarrow$ (i). Let $y \in T \mathscr{L}^{\prime}-\mathscr{L}^{\prime}$ and choose $x \in \mathscr{L}^{\prime}$ such that $T(x)=y$. Then there exists a sequence $\left\{y_{n}\right\}_{n=1}^{\infty}$ with distinct terms in $\mathscr{L}$ such that $\lim _{n} y_{n}=$ $x$. By continuity of $T, \lim _{n} T y_{n}=y$. Since $y \notin \mathscr{L}^{\prime}$, there exists a positive integer $N$ such that $T y_{n}=y$ for $n \geqq N$. It follows that $T^{-1} y$ is infinite, contradicting (iii).

TheOREM 2. $\mathscr{L}-\mathscr{L}^{\prime}$ is countable.

Proof. If $\mathscr{L}^{\prime}=\varnothing$, then $\mathscr{L}$ is finite, so suppose $\mathscr{L}^{\prime} \neq \varnothing$. For each positive integer $n$, there exist points $z_{1}, z_{2}, \cdots, z_{n_{k}}$ in $\mathscr{L}^{\prime}$ such that the open spheres, $S\left(z_{i}, 1 / n\right)$, of radius $1 / n$ about the points $z_{i}$ cover $\mathscr{L}^{\prime}$. For each $n$, let $A_{n}=\bigcup_{i=1}^{k_{n}} S\left(z_{i}, 1 / n\right)$ and note that each $\mathscr{L}-A_{n}$ is closed and disjoint from $\mathscr{L}^{\prime}$. It follows that $\mathscr{L}-A_{n}$ is finite for each $n$. It remains to show that $\mathscr{L}-\mathscr{L}^{\prime}$ is contained in the countable set $\bigcup_{n}\left(\mathscr{L}-A_{n}\right)$. But if $y \in \mathscr{L}-\mathscr{L}^{\prime}$, then for sufficiently large $n, 0<1 / n<d\left(y, \mathscr{L}^{\prime}\right)$, so that $y \in \mathscr{L}-A_{n}$. Thus $\mathscr{L}-\mathscr{L}^{\prime}$ is countable.

As shown by example (i), $\mathscr{L}-\mathscr{L}^{\prime}$ can be countably infinite when $\mathscr{L}^{\prime}$ is a proper subset of $T \mathscr{L}^{\prime}$. For the case where $\mathscr{L}-\mathscr{L}^{\prime}$ is infinite and $T \mathscr{L}^{\prime}=$ $\mathscr{L}^{\prime}$, see example (ii) of [1]. The following result shows that when $\mathscr{L}$ is countably infinite, then $\mathscr{L}-\mathscr{L}^{\prime}$ is always (countably) infinite.

THEOREM 3. If $\mathscr{L}^{\prime} \neq \varnothing$ and $\mathscr{L}-\mathscr{L}^{\prime}$ is finite (possibly empty), then $\mathscr{L}$ is uncountable.

Proof. Let $y \in \mathscr{L}^{\prime}$ and choose a sequence $\left\{y_{n}\right\}_{n=1}^{\infty}$ with distinct terms from $\mathscr{L}$ such that $\lim _{n} y_{n}=y$. Since $\mathscr{L}-\mathscr{L}^{\prime}$ is finite, $y_{n} \in \mathscr{L}^{\prime}$ for sufficiently large $n$. Hence $y \in\left(\mathscr{L}^{\prime}\right)^{\prime}$ and thus $\mathscr{L}^{\prime}$ is perfect and uncountable. Therefore $\mathscr{L}$ is uncountable.

We know of no example where $\mathscr{L}^{\prime} \neq 0$, and $\mathscr{L}-\mathscr{L}^{\prime}$ is nonempty and finite. Such an example may be difficult to construct as evidenced by Theorem 3. However, we do have the following result.

THEOREM 4. Suppose $\mathscr{L}^{\prime} \neq \varnothing$. The following statements are equivalent:

(i) $T \mathscr{L}^{\prime}-\mathscr{L}^{\prime}$ is finite.

(ii) $A=\left\{a \in \mathscr{L}^{\prime}: T a \notin \mathscr{L}^{\prime}\right\}$ is closed.

ProOF. (i) $\Rightarrow$ (ii). If $T \mathscr{L}^{\prime}-\mathscr{L}^{\prime}=\left\{y_{1}, y_{2}, \cdots, y_{n}\right\}$, then

$$
A=\bigcup_{i=1}^{n}\left(T^{-1} y_{i} \cap \mathscr{L}^{\prime}\right)
$$

is clearly closed since $T$ is continuous. 
(ii) $\Rightarrow$ (i). If $A$ is closed, then $T A=T \mathscr{L}^{\prime}-\mathscr{L}^{\prime}$, and $T A$ is closed. Thus if $T \mathscr{L}^{\prime}-\mathscr{L}^{\prime}=\left\{y_{n}: n \geqq 1\right\}$ is infinite, there is an infinite sequence $\left\{a_{n}\right\}$ of distinct terms in $A$ with $T a_{n}=y_{n}$ for each $n$. By compactness, it follows that $T A \cap \mathscr{L}^{\prime} \neq \varnothing$, a contradiction.

As exemplified by example (i) above, the set $A$ in Theorem 4 need not be finite.

4. Concluding remarks. The previous results give only partial answers to the following questions concerning the structure of $\mathscr{L}$ (for nonempty $\left.\mathscr{L}^{\prime}\right)$ :

1. Suppose $\mathscr{L}-\mathscr{L}^{\prime} \neq \varnothing$. Is $\mathscr{L}-\mathscr{L}^{\prime}$ infinite? Does $\mathscr{L}^{\prime}$ contain a fixed point of $T$ ?

2. What are necessary and sufficient conditions that $\mathscr{L}^{\prime}$ consist entirely of fixed points of $T$ ?

3. Can $T \mathscr{L}^{\prime}-\mathscr{L}^{\prime}$ be infinite?

By imposing further restrictions on $\mathscr{L}$ (e.g. $\mathscr{L}$ has a finite number of components) one obtains sufficient (but not necessary) conditions that $\mathscr{L}^{\prime}=T \mathscr{L}^{\prime}$. However, these conditions will not be considered here.

The authors wish to thank Mr. Anthony Biagioli for discussions concerning example (i) of this paper.

\section{REFERENCE}

1. F. T. Metcalf and T. D. Rogers, The cluster set of sequences of successive approximations, J. Math. Anal. Appl. 31 (1970), 206-212. MR 41 \#8743.

Department of Mathematics, Texas A\&M University, College Station, TEXAS 77843 